\title{
Actitudes lingüísticas de migrantes andinos en áreas sociodemográficas de lenguas en contacto
}

\author{
Linguistic Attitudes of Andean Migrants in \\ Sociodemographic Areas of Languages in Contact
}

\author{
Pedro Falcón Ccenta \\ Universidad Nacional Mayor de San Marcos, Lima, Perú \\ https://orcid.org/oooo-0002-0863-5735 \\ pfalconc@unmsm.edu.pe
}

Jorge Esquivel Villafana Universidad Nacional Mayor de San Marcos,Lima, Perú https://orcid.org/oooo-0oo1-7685-2742 jesquivelv@unmsm.edu.pe

\begin{abstract}
Resumen
Desde una perspectiva teórico-mentalista, se evaluó las identidades y las actitudes lingüísticas de los migrantes andinos en el asentamiento humano Villa Huanta, en San Juan de Lurigancho (Lima, Perú). Los enfoques cualitativo y cuantitativo posibilitaron la medición de las variables sexo, edad y escolaridad para lo cual se utilizó, de manera complementaria, el cuestionario, la observación participante y la entrevista semiestructurada. La evaluación cuantitativa permitió determinar que los migrantes andinos no evidencian una valoración muy alta hacia el castellano en relación con la lengua quechua, especialmente en el ámbito afectivo. Sin embargo, desde la perspectiva cualitativa, ello sí ocurre en los ámbitos cognoscitivos y socioculturales, no solo entre los jóvenes, sino también en la población adulta.
\end{abstract}

Palabras clave: Bilingües andinos; sociolingüística, sociodemografía lingüística, Lima, Perú.

\begin{abstract}
From a theoretical-mentalist perspective, identities, and linguistic attitudes of Andean migrants in the human settlement Villa Huanta, San Juan de Lurigancho (Lima, Peru) were evaluated. The qualitative and quantitative approaches allowed the measurement of the variables sex, age and schooling for which the questionnaire, participant observation and semi-structured interview were used in a complementary manner. A quantitative evaluation made it possible to determine that the Andean migrants did not show a very high value towards Spanish in relation to the Quechua language, especially in the affective sphere. However, from a qualitative perspective, this does occur in the cognitive and sociocultural spheres, not only among young people, but also in the adult population.
\end{abstract}

Keywords: Andean bilinguals; sociolinguistics, sociodemographic linguistics, Lima, Perú.

Recibido: 05-07-2020

Aprobado: 15-08-2020 
Actitudes lingüísticas de migrantes andinos en áreas sociodemográficas de lenguas... Pedro Falcón Ccenta y Jorge Esquivel Villafana

\section{Introducción}

La diversidad lingüística y la pluralidad cultural en el Perú generan procesos de interacción no igualitaria que desencadenan problemas y conflictos de orden lingüístico, educativo, social y cultural, los que demandan soluciones integrales acordes con la realidad particular de cada uno de los grupos existentes en el ámbito nacional. Por ello, el propósito del estudio es evaluar las actitudes lingüísticas en los ámbitos afectivos, cognoscitivos y socioculturales que predominan en los migrantes andinos del asentamiento humano Villa Huanta en el distrito de San Juan de Lurigancho, ubicado en el departamento de Lima.

Para la evaluación de las identidades y actitudes lingüísticas, se ha adoptado el enfoque mentalista (Agheyisi y Fishman, 1970; Fasold, 1996; Blas, 2012). Este enfoque considera la actitud como una disposición del hablante ante un estímulo, en este caso, la lengua. Respecto de los antecedentes, se encuentran, entre otros, los estudios de actitudes lingüísticas en comunidades bilingües de la selva central (Falcón, 2018a; Falcón, 2018b) y en la población asháninka (Falcón y Mamani, 2017). Otros trabajos han indagado las valoraciones de las variedades dialectales del español peruano (Arias, 2014). Sin embargo, son todavía escasos los trabajos sobre actitudes lingüísticas en el Perú, especialmente en poblaciones migrantes de Lima Metropolitana.

Con el auxilio de los instrumentos de recolección de datos, se describe los contextos comunicativos y las preferencias de lenguas, los cuales fueron correlacionados con las variables edad, sexo y escolaridad. Es pertinente señalar que las actitudes lingüísticas guardan estrecha relación con la construcción de la identidad. Al respecto, siguiendo a Howard (2007, p. 43), se distingue conceptualmente la identidad a nivel individual y a nivel colectivo, los que necesariamente están implicados, pues los conocimientos y experiencias del individuo cobran sentido en tanto sujeto involucrado en la red sociocultural. Por ello, el comportamiento actitudinal de los individuos y las funciones que tienen en la sociedad comprometen rasgos identitarios.

\section{MARCO TEÓRICO}

El enfoque mentalista, utilizado por investigadores contemporáneos (entre otros, Álvarez, 2001; Castillo, 2007; Arias, 2014; Zamora, 2015; Falcón y Mamani, 2017; Falcón, 2018b), con el apoyo de la psicología social, ha enfatizado el estudio de las actitudes con el fin de evaluar y dar cuenta del comportamiento actitudinal

80 Lengua \& Sociedad. Revista de Lingüística Teórica y Aplicada 
Actitudes lingüísticas de migrantes andinos en áreas sociodemográficas de lenguas...

Pedro Falcón Ccenta y Jorge Esquivel Villafana

de los individuos dentro de la sociedad. Cobra singular importancia comprender cómo, en ciertos casos, las personas expresan una valoración positiva hacia sus lenguas originarias y sus tradiciones, es decir, cómo manifiestan identificación con su legado, con su grupo. Sin embargo, la actuación cotidiana y espontánea no coincide necesariamente con lo antes señalado. No siempre existe una relación entre lo que dice el individuo respecto a la lengua y la cultura, y lo que realmente se evidencia en sus actuaciones cotidianas. Esta doble valoración para un mismo objeto se puede entender como una contradicción (Falcón, 2018a, 2018b y 2017). Por ello, se ha elegido el enfoque mentalista, ya que permite evaluar el proceso mental interno del individuo. En otras palabras, con el enfoque mentalista se realizan evaluaciones en diferentes contextos socioculturales, no solamente lo que las personas dicen, sino, además, quiénes lo dicen, cómo lo dicen, cuándo lo dicen y por qué lo dicen.

De esta manera, las actitudes lingüísticas son mediciones valorativas de las lenguas de convivencia, de los usuarios de estas lenguas, del comportamiento sociocultural que tienen y del proceso de construcción de identidades. De acuerdo con Álvarez (2001), la identidad supone una alteridad que bien podrían considerarse como las dos caras de una misma moneda, esto es, el vínculo estrecho entre las actitudes y los procesos de construcción de la identidad, en el entendido de que las actitudes condicionan la implementación de los procesos. Esta orientación posibilita incursionar en el análisis del proceso de valoración, es decir, cómo una persona percibe a los demás, cómo percibe la lengua, la cultura, los grupos diferentes.

En las investigaciones sociolingüísticas en el Perú, especialmente en migrantes amerindios, hay un vacío en cuanto a las actitudes hacia las lenguas originarias y el castellano, sea desde enfoques conductistas o mentalistas, y se desconoce acerca de las actitudes afectivas o cognoscitivas concomitantes con las identidades culturales. Por ello, respecto a los hechos sociolingüísticos que ejercen una influencia importante en las actitudes lingüísticas, el presente estudio podrá ser usado por otros investigadores como un prototipo para incursionar en el análisis e implementación de la teoría mentalista a fin de evaluar el uso y valoración de lenguas desde una óptica psicosociolingüística. En el escenario de la macrosociolingüística, posibilita la evaluación y la adecuación de los métodos utilizados en el actual contexto sociocultural de las comunidades bilingües y multidialectales. 
Actitudes lingüísticas de migrantes andinos en áreas sociodemográficas de lenguas... Pedro Falcón Ccenta y Jorge Esquivel Villafana

\section{Metodología}

En el marco de la metodología propuesta, de manera complementaria, se utilizaron los métodos directo e indirecto, cuyas técnicas fueron elaboradas en función de la realidad sociocultural de la zona a fin de desarrollar con claridad y objetividad un análisis cualitativo y cuantitativo de los datos.

El método directo generó la elaboración del cuestionario, instrumento que posibilitó la medición de las variables sexo, edad y escolaridad. El cuestionario elaborado constó de 39 preguntas, con respuestas de elecciones múltiples, referidas a las lenguas en contacto -la lengua quechua y la lengua castellana-, así como a la categorización sociocultural del asentamiento humano.

La metodología tuvo como soporte el diseño mixto, ya que se combinaron técnicas cuantitativas y cualitativas. Este tipo de método se enmarca en la tipología metodológica mixta (Hernández et al., 2010, p. 553).

Específicamente, el diseño es transeccional porque se trabajó en un tiempo único y es correlacional porque se circunscribió a correlacionar las variables de investigación, sin pretender establecer relaciones de causa-efecto.

Con respecto a los instrumentos y su aplicación, los datos cuantitativos se procesaron en hojas de cálculo de Excel para poderlos exportar al programa SPSs. Consecuentemente, se buscó determinar las correlaciones correspondientes acorde con los objetivos del estudio. Considerando los datos registrados a través de la observación participativa, se evaluaron las actitudes de los participantes respecto de la lengua quechua y del castellano. Para ello, complementariamente se organizó la observación en un cuadro codificado por familias, que se estructuró tomando como referencia cinco ejes, los que resumimos en tres:

a. Actitudes naturales hacia el castellano y hacia la lengua quechua en el comportamiento de la vida diaria, puntualizando las variables contempladas en el estudio (edad, sexo, escolaridad).

b. Contextos y usos del castellano y del quechua en la cotidianeidad: intrafamiliar, interfamiliar, comunal e interacción con foráneos.

c. Valoración de la lengua quechua en el desarrollo de actividades cotidianas.

82 Lengua \& Sociedad. Revista de Lingüística Teórica y Aplicada 


\section{POBLACIÓN Y MUESTRA}

La población estuvo compuesta por 191 personas entre varones y mujeres. Como criterios de evaluación de la muestra se tomó en cuenta las variables sociolingüísticas de sexo, escolaridad y edad.

El estudio se desarrolló en el AA. HH. Villa Huanta (San Juan de Lurigancho, Lima), que tiene una población aproximada de 1500 habitantes. Se realizó un censo poblacional en el que se contabilizaron 532 mujeres y 529 varones, de acuerdo con la información que nos proporcionaron las familias. Muchas casas no fueron censadas por ausencia de los moradores o porque se negaron a ser censados. El registro censal se efectuó por solicitud de los dirigentes del AA. HH., como parte de una iniciativa de responsabilidad social.

\section{Interpretación y discusión de los resultados}

Se presenta el análisis estadístico de los datos registrados en la encuesta efectuada en Villa Huanta, conformada básicamente por migrantes andinos bilingües del distrito de Huanta, provincia de Ayacucho. La evaluación cuantitativa se correlacionó con el análisis cualitativo producto de las entrevistas y de la observación participante.

\subsection{Datos generales}

La comunidad de Villa Huanta, ubicada en el distrito de San Juan de Lurigancho, está conformada básicamente por migrantes bilingües que llegaron a Lima procedentes de Ayacucho, zona andina enclavada en el sur del Perú. Su situación lingüística corresponde a una zona de bilingüismo social desequilibrado, donde el castellano es considerado como la lengua de prestigio, en desmedro del disminuido valor social que representa el quechua.

Para la ejecución de la investigación relativa a la identificación y descripción de los rasgos identitarios y las actitudes lingüísticas de los pobladores de la comunidad de Villa Huanta, se elaboró un cuestionario de 39 preguntas correspondientes a tres aspectos temáticos: contactos sociales, uso de lenguas y valoración de las lenguas. Para este artículo, por razones de espacio, se han tenido en cuenta solo las preguntas consideradas más relevantes, a fin de no resentir el propósito y los contenidos medulares de la investigación. 
Actitudes lingüísticas de migrantes andinos en áreas sociodemográficas de lenguas... Pedro Falcón Ccenta y Jorge Esquivel Villafana

A continuación, las tablas 1 y 2 presentan información desagregada por grupos de edad y por escolaridad.

Tabla 1. Grupos etarios

\begin{tabular}{l|l|l}
\hline Edad & Frecuencia & Porcentaje \\
\hline 18 a 30 años & 15 & $8 \%$ \\
\hline 31 a 45 años & 27 & $14 \%$ \\
\hline 46 a 60 años & 78 & $41 \%$ \\
\hline 61 a más años & 71 & $37 \%$ \\
\hline Total & 191 & $100 \%$ \\
\hline
\end{tabular}

Tabla 2. Grados de escolaridad

\begin{tabular}{l|l|l}
\hline Escolaridad & Frecuencia & Porcentaje \\
\hline Primaria & 25 & $13 \%$ \\
\hline Secundaria & 82 & $43 \%$ \\
\hline Superior & 79 & $41 \%$ \\
\hline Ninguno & 5 & $3 \%$ \\
\hline Total & 191 & $100 \%$ \\
\hline
\end{tabular}

\subsection{Contactos sociales}

A pesar de que la mayoría del grupo es bilingüe quechua-castellano, en ese orden de adquisición de las lenguas, la lengua que se emplea más frecuentemente en la comunidad es el castellano ( $71 \%$ ), le sigue el uso alternado de las lenguas quechua-castellano (16\%). Son pocos los que tienen la impresión de que su lengua materna, el quechua, es la que más se habla en la comunidad (7 \%).

\subsection{Empleo del quechua}

En la comunidad hay una clara situación de bilingüismo diglósico, pues el castellano, dado su considerable prestigio social, es empleado en toda la comunidad y en situaciones comunicativas con los vecinos, autoridades, familiares, incluso en la casa con los hijos menores y jóvenes, quienes son monolingües 
Actitudes lingüísticas de migrantes andinos en áreas sociodemográficas de lenguas...

Pedro Falcón Ccenta y Jorge Esquivel Villafana

castellanohablantes. También ocurre esta situación en la escuela, donde la comunicación es exclusivamente en castellano.

En cambio, debido a su disminuido estatus social, se reserva el empleo del quechua para situaciones comunicativas muy restringidas con los vecinos (21\%), en la casa, en el entorno familiar íntimo, con los padres (11\%) y hermanos mayores (6\%) y con otras personas con quienes, por el nivel de confianza, se intercambia el discurso quechua (18\%); con nadie (3\%).

Como se advierte, hubo entrevistados bilingües que manifestaron no hablar quechua con nadie (3\%), probablemente con el propósito de pasar inadvertidos e identificarse con la lengua castellana exclusivamente, dada su alta consideración social. Detrás del sentimiento negativo hacia la lengua nativa y, por ende, hacia el grupo étnico, se advierte una clara situación de bilingüismo encubierto (Blas, 2012, p. 352).

Cabe recalcar que en la percepción del factor identitario no es suficiente el hecho de hablar castellano. También cumple un papel importante la forma de hablarlo. Para evitar ser identificado como bilingüe, el habla castellana debe estar libre de la influencia fonémica del quechua (Howard, 2007, p. 58).

\subsection{Uso de la lengua según la comunidad de origen}

Con respecto a la utilización del quechua o el castellano en la comunidad de origen, la mayoría de los entrevistados (73 \%) refirió que habla quechua en su pueblo de origen; $13 \%$ no lo habla ni en su pueblo de origen; el $14 \%$ permaneció callado a la pregunta. En cuanto al empleo del castellano en la comunidad de origen, el $58 \%$ refirió positivamente. Viajar al lugar de origen y hablar la lengua quechua en el contexto nativo logra mantener el sentimiento de fidelidad al grupo étnico; hablar el quechua consolida la relación entre la identidad lingüística y la identidad territorial (Gugenberger, 1999). Los datos presentados indican que en la comunidad de origen se habla las dos lenguas: castellano y quechua con cierta preferencia por el quechua; la elección probablemente dependa del tipo de interlocutor y del nivel de confianza entre los coterráneos. De todos modos, en la decisión de utilizar el quechua, se compromete cierta dosis de lealtad con la lengua nativa.

\subsection{Uso de lenguas}

En cuanto a la pregunta acerca de la lengua que se emplea en la comunicación diaria (tabla 3), una mayoría significativa refirió que emplea el castellano (71\%); 
Actitudes lingüísticas de migrantes andinos en áreas sociodemográficas de lenguas... Pedro Falcón Ccenta y Jorge Esquivel Villafana

un escaso número de personas admitió que emplea el quechua (4\%); el porcentaje de personas que emplea el quechua y el castellano alternadamente fue de $23 \%$.

Tabla 3. Uso de lenguas

\begin{tabular}{l|l|l}
\hline $\begin{array}{l}\text { ¿Qué lengua empleas en tu } \\
\text { comunicación diaria? }\end{array}$ & Frecuencia & Porcentaje \\
\hline Quechua & 7 & $4 \%$ \\
\hline Castellano & 136 & $71 \%$ \\
\hline Quechua y castellano & 43 & $23 \%$ \\
\hline No responde & 5 & $3 \%$ \\
\hline Total general & 191 & $100 \%$ \\
\hline
\end{tabular}

Cabe precisar que la alternancia, en este caso, no se debe a la simple decisión de emplear indistintamente una u otra lengua en la comunicación diaria, sino que ella está condicionada por factores extralingüísticos como el lugar o el contexto situacional, si el interlocutor es una autoridad local o vecino de confianza, el momento, el tema y otras circunstancias que se vinculan con el esquema actitudinal diglósico y la relación de valor social. Los usos idiomáticos están asociados a la presión social. El escaso valor social del quechua y el sentimiento de inferioridad asociado afectan su escasa preferencia de uso, sobre todo en la ciudad, donde no puede competir con el predominio de la lengua oficial.

\subsection{Valoración de las lenguas}

En lo que se refiere a la valoración afectiva de las lenguas castellano y quechua, el $60 \%$ refirió que la lengua quechua tiene sonidos más agradables con respecto a la lengua castellana (9\%); el $31 \%$ opinó que ambas. Esto es un fuerte indicio del nivel de afectividad que el bilingüe le dispensa a la lengua materna.

A pesar de hallarse en un contexto ajeno al lugar de origen, el quechua hablante mantiene una actitud favorable con respecto a su lengua materna. Al margen del escaso valor instrumental del quechua, la mayoría de los entrevistados la valora emocionalmente. Este sentimiento de lealtad hacia la lengua minoritaria en un contexto de diglosia desfavorable a ella revela el conflicto sociolingüístico que enfrentan los quechua hablantes, quienes, por un lado, buscan el interesado control de la lengua castellana por su valor social; y por otro, mantienen el sentimiento 
Actitudes lingüísticas de migrantes andinos en áreas sociodemográficas de lenguas...

Pedro Falcón Ccenta y Jorge Esquivel Villafana

afectivo hacia su lengua y su cultura. Un grupo minoritario (31\%) estimó que ambas lenguas tienen sonidos agradables.

Cuando se trata de responder a la pregunta: ¿En qué lengua puedes expresar mejor lo que piensas y lo que sientes?, aflora un apego a la lengua predominante en el contexto y los hablantes manifiestan que su mayor competencia para expresar lo que piensan y lo que sienten lo muestran con el empleo del castellano (50 \%); otro grupo ligeramente menor opta por ambas lenguas (40 \%) y un grupo minoritario (10 \%) mantiene su sentimiento de lealtad hacia la lengua quechua.

\subsection{Valoración del quechua}

En cuanto a la facilidad o dificultad para escribir la lengua quechua en relación con el factor utilitario, la mayoría de los encuestados consideró que la lengua quechua no es fácil de escribir, pero es útil (32\%). Otro grupo mayoritario (25\%) opinó que ella es fácil de escribir, además de ser útil; un escaso $3 \%$ opinó que la lengua quechua no es fácil de escribir ni es útil.

Tabla 4. Valoración del quechua

\begin{tabular}{l|l|l}
\hline La lengua quechua & Frecuencia & Porcentaje \\
\hline Es fácil de escribir & 19 & $10 \%$ \\
\hline No es fácil de escribir & 26 & $14 \%$ \\
\hline No es útil & 2 & $1 \%$ \\
\hline Es útil & 30 & $16 \%$ \\
\hline Es fácil de escribir y es útil & 48 & $25 \%$ \\
\hline No es fácil de escribir y no es útil & 5 & $3 \%$ \\
\hline No es fácil de escribir, pero es útil & 61 & $32 \%$ \\
\hline Total general & 191 & $100 \%$ \\
\hline & &
\end{tabular}

Como se aprecia, no hay consenso acerca de la facilidad o dificultad para la escritura del quechua. Esto puede deberse a la escasa promoción de su escritura en los ámbitos escolar y universitario, pero se reconoce la utilidad o el valor funcional del quechua en sus funciones comunicativa y social dentro de la comunidad, por lo que los encuestados la valoraron significativamente con un acumulado de (57\%) en su totalidad. Ella pervive afectivamente en la conciencia de los hablantes. En 
Actitudes lingüísticas de migrantes andinos en áreas sociodemográficas de lenguas... Pedro Falcón Ccenta y Jorge Esquivel Villafana

situaciones de conflicto despertadas por la exigencia de una impresión valorativa con respecto a la lengua quechua en la comunidad, la respuesta fue siempre favorable a la lengua nativa, depositaria del orgullo del grupo étnico.

\subsection{Valoración del castellano}

Las preguntas relativas a la valoración del castellano (tabla 5) suscitaron respuestas contundentes y compatibles con una situación de internalización de la ideología diglósica.

\section{Tabla 5 Valoración del castellano}

\begin{tabular}{l|l|l}
\hline La lengua castellana es & Frecuencia & Porcentaje \\
\hline Difícil de aprender & 20 & $10 \%$ \\
\hline Fácil de aprender & 152 & $80 \%$ \\
\hline Ni fácil ni difícil de aprender & 19 & $10 \%$ \\
\hline Muy difícil de aprender & 0 & $0 \%$ \\
\hline Total general & 191 & $100 \%$ \\
\hline
\end{tabular}

La valoración positiva del castellano en una comunidad bilingüe tiene que ver con los valores y prejuicios imperantes. El 80 \% opinó que la lengua castellana es fácil de aprender. Ninguno consideró su posible dificultad (o \%). Estos resultados van de la mano con los valores dominantes en la sociedad, donde la «lengua castellana es la lengua mejor valorada por la enorme mayoría de personas y las lenguas originarias del Perú son consideradas inferiores» (Pérez, 2004, p. 74). La valoración positiva recae en el castellano: es una lengua formal, de la ciudad, con acceso escolar para su aprendizaje y de proyección mundial.

\subsection{Importancia y uso de las lenguas}

Acerca de la importancia y uso de las lenguas quechua y castellana, el análisis de los resultados muestra que cuando se relacionan las variables importancia y uso de la lengua, las respuestas reiteraron la evidencia de una situación de identidad en conflicto. Esto se debe a que, si bien expresan respuestas deseables con verbos conjugados en condicional (debería), su comportamiento y conducta idiomática vigentes están polarizados, pues muestran lo contrario. 
Actitudes lingüísticas de migrantes andinos en áreas sociodemográficas de lenguas...

Pedro Falcón Ccenta y Jorge Esquivel Villafana

Las preguntas referidas en este punto aluden a situaciones deseables, futuristas, a un «deber ser», que generan respuestas que en la práctica colisionan con actitudes y conductas contradictorias. Así, expresaron que «todas las personan andinas deberían saber la lengua quechua (95\%) y también la lengua castellana (91 \%)», aunque en la práctica no enseñan la lengua quechua a sus familiares; que la lengua quechua debería emplearse en la escuela (98\%), en las redes sociales (95\%), en los periódicos y revistas (96\%) y en la radio y televisión (97\%).

Todas esas expresiones colisionan con la realidad, pues el quechua solo se usa en el contexto familiar o con los vecinos de confianza. Su uso se limita siempre a personas mayores de edad por temor a la exclusión social. En esas conductas, se percibe un desdoblamiento de la conciencia lingüística del hablante, que cuando expresa respuestas a situaciones anheladas en el futuro asume una identidad proquechua, aunque en la práctica asuma una identidad negativa con respecto a la lengua aborigen. Howard (2007, p. 78) asegura que «existe una dimensión metadiscursiva de estos intercambios que reflexionan sobre la realidad más que vivirla», y refiere el hecho contrastante de que incluso los profesionales bilingües que hablan una lengua indígena como lengua materna y trabajan en el área de la educación bilingüe intercultural no trasmiten la lengua a sus hijos.

\section{Conclusiones}

Se reconoce una clara situación de ideología diglósica en la comunidad estudiada, pues el castellano es considerado como la lengua de mayor uso, que abarca casi toda situación comunicativa. En cambio, el empleo del quechua está restringido a contextos familiares básicamente y al trato con vecinos con los que se mantiene cierto nivel de confianza. No escapan situaciones de bilingüismo encubierto, donde algunos bilingües niegan y reniegan de la lengua materna con el claro propósito de despojarse del estigma social que ella representa. Así, trasladan su preferencia lingüística hacia la lengua castellana exclusivamente, porque representa los valores positivos imperantes.

La mayoría de los entrevistados refirió viajar a su pueblo de origen y hablar el quechua con sus paisanos, con quienes rememora la relación de identidad lingüística y territorial. Los bilingües otorgaron un fuerte nivel de afectividad a la lengua quechua, lo cual sugiere el desarrollado sentimiento de lealtad hacia la lengua nativa en un contexto de diglosia desfavorable a ella. La lengua castellana, 
Actitudes lingüísticas de migrantes andinos en áreas sociodemográficas de lenguas... Pedro Falcón Ccenta y Jorge Esquivel Villafana

en cambio, recibió una aceptación contundente desde la perspectiva de su valor funcional y social.

Las entrevistas dejaron entrever situaciones de conflicto por parte del entrevistado, quien por un lado expresa juicios y afirmaciones favorables al quechua con la finalidad de satisfacer las expectativas del entrevistador y, por otro, en la práctica, asume una conducta negativa con respecto a la lengua quechua. Las actitudes lingüísticas vinculadas con la identidad del grupo en el asentamiento humano Villa Huanta evidencian, en términos generales, sobre todo en la población joven, rasgos de identidad de grupo bastante débiles.

Las variables sexo y edad, si bien cuantitativamente no tuvieron mayor influencia en la valoración de las lenguas originaria y castellana, cualitativamente sí se observó una mayor valoración cognoscitiva y sociocultural hacia la lengua castellana por parte de los jóvenes, incluso en el ámbito afectivo.

Los contextos comunicativos de la lengua quechua son menos funcionales que los del castellano debido a la naturaleza instrumental de mayor alcance de este. Si bien ambas lenguas adquieren capital importancia entre los usuarios indígenas, especialmente por parte de los mayores, la valoración afectiva los conduce innegablemente a la identificación con la lengua originaria; sin embargo, en cuanto a las acciones pragmáticas, para los migrantes andinos usuarios, de la lengua quechua, el castellano cobra mayor ponderación por su estatus y prestigio.

\section{Referencias bibliográficas}

Agheyisi, R. y Fishman, J. (1970). Language attitude studies: A brief survey of methodological approaches. Anthropological Linguistics, 12, 137-157.

Álvarez, A. (2001). Actitudes lingüísticas en Mérida y Maracaibo: otra cara de la identidad. Boletín Antropológico, 2(52), 145-166.

Arias, A. (2014). Actitudes lingüísticas en el Perú. Predominancia del castellano de la costa central y norte. Bergen Language and Linguistic Studies, 5, 1185-1248. Disponible en: https://bells.uib.no/index.php/bells/article/view/692

Castillo, M. (2007). Mismo mexicano pero diferente idioma. Identidades y actitudes lingüísticas en los maseualmej de Cuetzalán. México D. F.: Instituto Nacional de Antropología e Historia, Universidad Nacional Autónoma de México.

Falcón, P. (2018a). Identidades y preferencias lingüísticas en comunidades de la selva central. Revista Letras, 89(129), 128-153. 
Actitudes lingüísticas de migrantes andinos en áreas sociodemográficas de lenguas... Pedro Falcón Ccenta y Jorge Esquivel Villafana

Falcón, P. (2018b). Identidades y actitudes lingüísticas en comunidades bilingües de la selva central. Lima: Universidad Nacional Mayor de San Marcos

Falcón, P. y Mamani, L. (2017). Actitudes lingüísticas en contextos interculturales: Población ashaninka Bajo Chirani. Revista de Lingüistica Teórica y Aplicada, 55(1), 95-115.

Fasold, R. (1996). La sociolingüística de la sociedad. Madrid: Visor Libros.

Hernández, R., Fernández, C. y Baptista, M. (2014). Metodología de la investigación. México D. F., México: McGraw Hill.

Howard, R. (2007). Por los linderos de la lengua. Lima: Pontificia Universidad Católica del Perú.

Pérez, J. (2004). Los castellanos del Perú. Lima: Programa de Educación Básica de la Cooperación Alemana al Desarrollo proeduCA-GTZ.

Zamora, Z. (2015). Las actitudes lingüisticas en Nicaragua. Lo que pensamos los nicaragüenses sobre el español que hablamos. Nicaragua: PAVsA. 